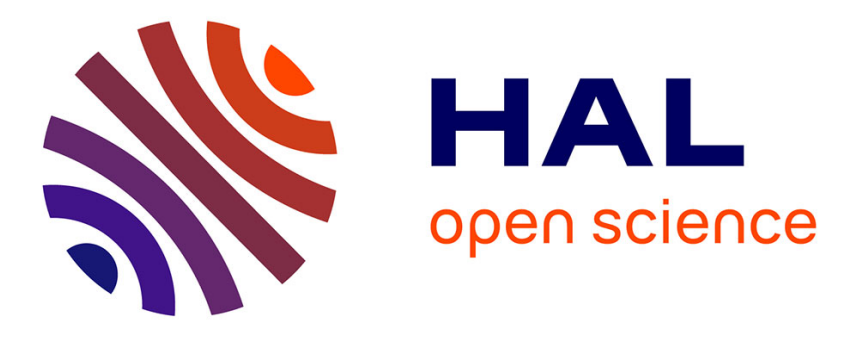

\title{
Game-Based Adaptive Remote Access Vpn for Iot: Application to E-Health
}

Amel Arfaoui, Ali Kribèche, Sidi-Mohammed Senouci, Mohamed Hamdi

\section{To cite this version:}

Amel Arfaoui, Ali Kribèche, Sidi-Mohammed Senouci, Mohamed Hamdi. Game-Based Adaptive Remote Access Vpn for Iot: Application to E-Health. 2018 IEEE Global Communications Conference (GLOBECOM), Dec 2018, Abu Dhabi, United Arab Emirates. 10.1109/GLOCOM.2018.8648064 . hal-02557007

\section{HAL Id: hal-02557007 https://hal.science/hal-02557007}

Submitted on 14 Feb 2022

HAL is a multi-disciplinary open access archive for the deposit and dissemination of scientific research documents, whether they are published or not. The documents may come from teaching and research institutions in France or abroad, or from public or private research centers.
L'archive ouverte pluridisciplinaire HAL, est destinée au dépôt et à la diffusion de documents scientifiques de niveau recherche, publiés ou non, émanant des établissements d'enseignement et de recherche français ou étrangers, des laboratoires publics ou privés.

\section{다)(1) $(5$}

Distributed under a Creative Commons Attribution - NonCommerciall 4.0 International 


\title{
Game-Based Adaptive Remote Access VPN for IoT: Application to e-Health
}

\author{
Amel Arfaoui**, Ali Kribecheł, Sidi Mohammed Senoucił, Mohamed Hamdi* \\ ${ }^{\ddagger}$ DRIVE EA1859, Univ. Bourgogne Franche Comté, France \\ *Digital Security Unit, SupCom University of Carthage, Tunisia \\ \{amel.arfaoui, Sidi-Mohammed.Senouci,Ali.Kribeche01\}@u-bourgogne.fr,mmh@supcom.rnu.tn
}

\begin{abstract}
Internet of Things (IoT) based pervasive healthcare systems have revolutionized the healthcare industry while enabling remote patient monitoring to improve patient care delivery and provide a highly reliable ubiquitous healthcare monitoring. In this context, Virtual Private Network (VPN) is a promising network layer technology that is used for a secure, reliable and remote access to patient health information. It is an overlay network that creates secure and dynamic tunnels between various devices across a public network such as the Internet. However, it is vulnerable to several attacks such as spoofing, snipping, and hacking as the data is transmitted through the Internet connection. In addition, due to the IoT device's energy constraints and the application requirements, the main purpose is to adaptively select the most appropriate encryption and authentication algorithms to secure the communication tunnel between these resource constrained devices and the remote user. Therefore, adaptive risk-aware secure tunnel negotiation is perceived as a major performance objective. In this paper, we propose a Stackelberg game for the security requirements negotiation between the communication peers in order to ensure a trade-off between security effectiveness and network performance while considering the dynamic context changes. Simulation results prove that the proposed approach can reduce the cost of security policy implementation in terms of performance degradation. For instance, the latency is improved by around $25 \%$ compared to static security policy.
\end{abstract}

Keywords-, IoT, VPN, Tunnel, Negotiation, Game theory, Nash Equilibrium, Adaptive security.

\section{INTRODUCTION}

Remote patient monitoring has emerged as a pioneering solution that redefines the way of healthcare access and delivery. It provides medical access convenience, real-time monitoring, personalized clinical diagnosis, and chronic disease treatment. However, the open nature of wireless communication makes the transmitted data prone to several security threats such as spoofing, modification, and eavesdropping. Therefore, in a dynamic environment such as IoT where a variety of data should be continuously exchanged and accurately processed on time, security and network performance are primary requirements that need a special attention while considering the IoT devices' limited resources and context changes.

In this context, Virtual Private Network (VPN) technology is considered as the first line of defense for IoT-based healthcare systems. It is widely deployed to provide security services such as confidentiality, integrity, and availability over insecure public networks. It allows a private and secure tunnel to be created between the communicating parties in order to protect the exchanged information against false data origins as well as passive and active intruders. In fact, the tunnel creation process requires firstly the establishment of a preparatory encrypted and secure tunnel, then from within that channel, the encryption keys and parameters are negotiated. On the one hand, Internet Security Association and Key Management Protocol (ISAKMP) is used to manage keys and Security Associations (SA) that define the security attributes and authenticate the communication peers' identities. On the other hand, Internet Key Exchange (IKE) is defined for negotiating the connection to authenticate both peers of the secure tunnel by exchanging shared keys [1]. However, the deployment of VPN in the IoT environment still faces many security and QoS challenges to be overcome. In fact, harsh environmental conditions and stringent resource constraints of IoT devices (energy consumption, computational and memory capacity as well as communication bandwidth) [2] make security costs in terms of energy depletion and performance degradation more burdensome. In addition, in a dynamic IoT environment, static security policy is inefficient since it doesn't consider the dynamic context changes that involves the traffic nature, the devices' capabilities as well as the QoS requirements. Furthermore, existing VPN approaches [3][4][5] do not incorporate the contextual information to adaptively negotiate the security attributes that include encryption protocols (DES, 3DES, and AES), integrity protocols (MD5 and SHA), and the key length (768-bit, 1024-bit, 1536-bit).

In order to address the above issues, an adaptive risk assessment model that reflects the characteristics of the IoT environment and identifies the most suitable security policy is needed. For this purpose, we propose a novel Stackelberg game for adaptive security policy negotiation between the communicating parties on the basis of the risk score in order to choose the optimal VPN configuration while considering the dynamic context changes. The proposed approach requires continuous monitoring and analysis of the application requirements and the devices' capabilities to select the right security attributes on the basis of the security risk. The novelties of our proposed model consist of:

- An adaptive risk assessment framework for IoT communication that is designed to estimate and predict the risk score in order to select the most appropriate VPN configuration and assess its impact on the network performance, 
- A dynamic context model for adaptive security management that represents the core of the security policy decision-making,

- A novel game-theoretic approach to model the adaptive and context-aware negotiation of the security policy in order to choose the appropriate cryptographic primitives and thus, ensure a trade-off between security effectiveness and network performance.

The rest of the paper is organized as follows. Section II discusses some previous works related to adaptive security management. In Section III, we present the system model. In Section IV, we propose an adaptive risk assessment framework. A Stacklberg game for adaptive security policy negotiation on the basis of the risk score is formulated in Section V. Numerical simulation results are given in Section VI. Finally, Section VII concludes the paper.

\section{RELATED WORK}

VPN has drawn a significant attention as a cost-effective solution for ubiquitous, continuous and flexible remote control. However, its integration in the IoT environment is considered as a challenging job that should consider data scalability, reliability, security effectiveness, QoS requirements and resource-constrained devices. In this context, security functions that consist of data encryption and authentication, and security policy negotiation should be defined while taking into account its impact on the network performance. Recently, some contributions have addressed the performance of VPN and outlined the necessity of adaptive security management. In [3], authors analyzed the network performance of site-to-site (GRE and IPSec) and remote access (PPTP and L2TP) VPN protocols on the basis of the various application requirements. The protocols performances are evaluated in terms of throughput, RTT, jitter and security parameters. In [4], authors addressed the performance evaluation of Secure Socket Tunneling Protocol (SSTP) and Internet Key Exchange Version 2 (IKEv2) VPN protocols. Based on a test-bed environment where VPN clients exchange data, it was found that IKEv2 is more efficient than SSTP in terms of throughput, jitter, and delay. In [5], a patent proposed Dynamic Group VPN (DGVPN) approach that used a unique key for data encryption and decryption of the same gateway during each session established between two gateways. The proposed model handles the principle of security group where a set of gateways may share the same security associations. However, it lacks scalability given that the same VPN router should be associated with several groups to profit from different security levels. The invention patent [6] developed a VPN architecture to manage partitioned access VPNs on multiple domains. Even if a high security level is provided, the network performance isn't considered given that higher security level induces heavy processing time for sensitive-delay application.

To respond to the limitations of the above contributions, [1] proposed a novel adaptive security model for New Generation VPN. The proposed model provides an intelligent and adaptive security decision while considering the performance requirements of the transported traffic. Specifically, for a sensitive-delay application, if the network is overloaded then the security level should be reduced to medium, low or even not if the organization accepts it. However, this approach didn't incorporate the adaptive negotiation of the security policy or the risk score associated with the communication on the basis of the contextual information. In [7], authors proposed a game-based adaptive security model for IoT-eHealth application. They mainly focused on the smart things dynamic environment and capabilities in order to determine whether or not messages authentication should be enabled and thus ensure a tradeoff between energy efficiency and security effectiveness. In [8], a stochastic game for adaptive security has been presented to achieve a balance between network performance and security level while taking into account the contextual information that involves the application requirements, the IoT environment as well as devices capabilities. However, none of the aforementioned works has introduced the VPN in the IoT environment or incorporated the dynamic context changes into the negotiation process for secure tunnel establishment between the communicating parties. Therefore, the main idea of this study is to design an adaptive security management approach that determines the suitable security policy according to the dynamic context changes including the IoT device's capabilities, the application requirements (latency, throughput, packet loss) as well as the traffic nature and load. For instance, if the network performance is unacceptable with the highest security settings, it is better to degrade the security level to achieve the desired network performance.

\section{SYSTEM MODEL}

In this section, we present the system model that we have adopted to implement the adaptive security negotiation for secure tunnel creation. We consider the remote patient monitoring system where healthcare providers may remotely and securely access to IoT devices through a gateway. At first, we present the remote access control model that defines the interactions between the different entities involved into the security policy selection. Then, we discuss the VPN tunnel creation process and the negotiation between the communication peers.

\section{A. Remote Access VPN Model}

To handle a remote user access to a smart healthcare space protected by an IoT gateway, a VPN tunnel establishment process is launched and an IKE (Internet Key Exchange) negotiation is initiated between the peers. As shown in Figure 1, the remote user initiates a connection to the IoT gateway that has the role of adapting the communication between the IoT devices and the remote user. The user management is performed through the central server belonging to VPN Site 2. In order to define a suitable security policy to be delivered to the remote user, the negotiation of the security parameters in terms of authentication, encryption and key length between the communicating parties should consider the risk score and its impact on the network performance. In this context, the IoT gateway verifies the user profile by querying the central server behind the security gateway in the VPN site 2 . In addition, it defines the security level supported by the IoT device on the basis of its capabilities in order to achieve a tradeoff between the security level and the network performance. 


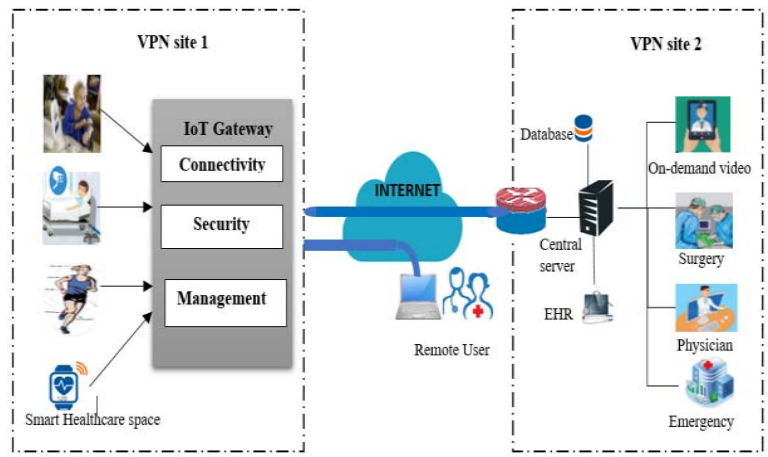

Figure 1. VPN tunnel establishment for remote patient monitoring

\section{B. End-to end secure communication channel establishment}

We consider the establishment of an end-to-end secure communication channel between an IoT device and a remote user. For this purpose, a trusted gateway is used to relieve the IoT device from heavy cryptographic tasks. We notice that the IoT device is authenticated with the gateway and a secure communication channel is established between them.

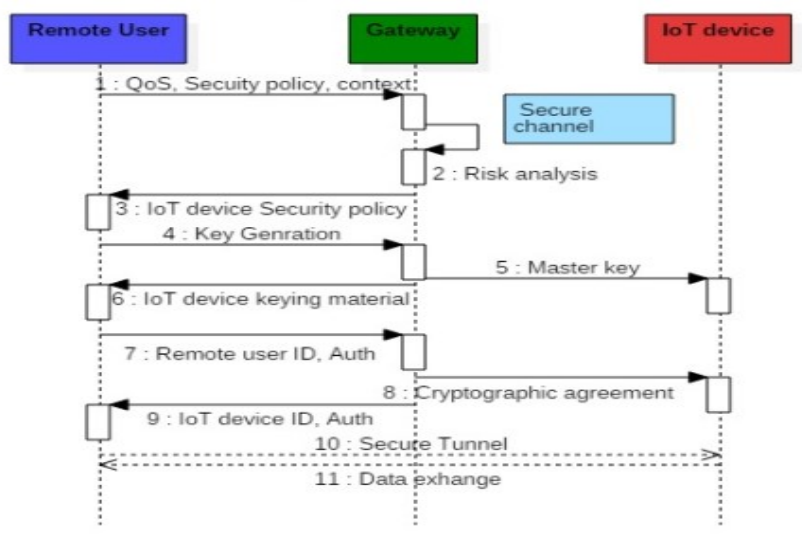

Figure 2. Secure tunnel establishment negotiation

As shown in Figure 2, in the first exchange between the communication peers, the remote user initiates a service request to the IoT device, including the desired security level, the QoS requirements as well as the contextual information. The gateway intercepts the message and plays the role of the IoT device. Specifically, it negotiates the security primitives on behalf of the IoT device while taking into account the device's capabilities and the risk score associated with the requested service, by sending a message which contains a valid security policy supported by the constrained IoT device. As a second exchange, the remote user generates the key for the establishment of the ISAKMP security association and forwards it to the gateway. Upon receiving the keying material message, the gateway generates the key on behalf of the smart thing. Then, it dispatches the master key to the constrained IoT device through a pre-established secure channel. Once the key exchange phase is accomplished, the authentication credentials are exchanged between the remote user and the gateway through an encrypted channel that uses the shared secret key.

\section{ADAPTIVE RisK ASSESSMENT FOR REMOTE ACCESS VPN}

In the proposed model, the risk analysis (as presented in Figure 3) is required for the VPN tunnel negotiation in order to ensure a tradeoff between security effectiveness and network performance. In fact, the adopted security policy should define the most appropriate security parameters in terms of authentication, encryption algorithms as well as the encryption key length on the basis of the risk score.

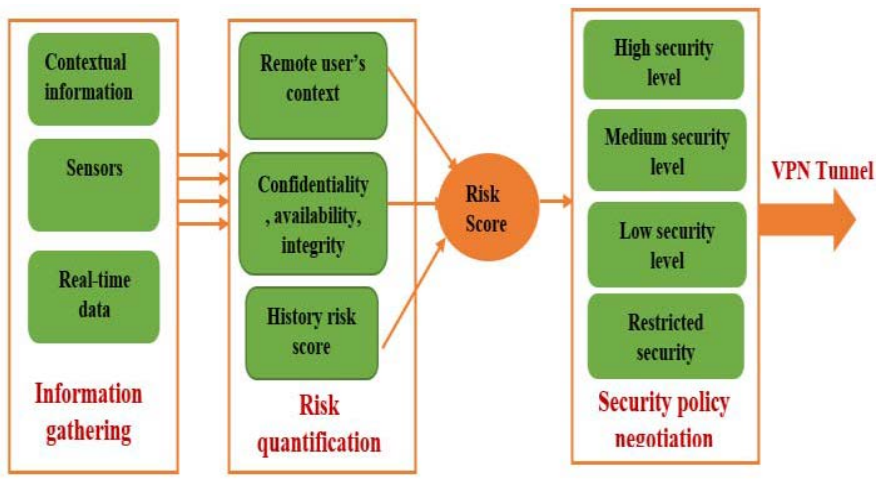

Figure 3. Context-aware risk management framework

The different components of the adaptive risk assessment framework are presented as follows:

- Information gathering: This phase collects the contextual information associated with the service request in order to evaluate the risk level. In fact, it conducts a continuous monitoring of the requester environment as well as the IoT device's capabilities to quantify the risk score and choose the required security level.

- Risk quantification: This phase incorporates different factors that affect the risk level. Particularly, it considers the data flow nature, load, and type, the remote user's credentials, the impact of the access request on confidentiality, integrity, and availability as well as the historical risk information. Once the risk score is determined, the negotiation process is initiated between the communicating parties to establish a secure tunnel. In this context, we propose a Stackelberg game (defined in Section V) to present the security primitives negotiation while considering the dynamic context changes.

- Security policy negotiation: In this phase, the suitable security policy is defined on the basis of the risk level. Specifically, the security parameters (such as keys, authentication and encryption algorithms) are negotiated. The generic policies adopted in the proposed approach are: High Security level, Medium security level, Low security level and restricted. The first two policies guarantee the confidentiality, authentication, and integrity, the third one supports the integrity and data authentication. The latest policy prioritizes the network performance where no security control is performed for the transported data. 
In the following, we define the different factors that should be involved to estimate the risk level. Particularly, we consider the contextual information, $c$, the security characteristics of access privileges, $a$, on a resource $o$ as well as the metric related to the history of the user's actions.

\section{A. Context-awareness modeling}

In a healthcare system, medical data should be communicated securely to the well-authenticated parties while considering the dynamic context changes. In this context, VPN security parameters should be negotiated between the peers on the basis of the dynamic context changes. Particularly, the negotiation process is based on risk quantification that considers not only the user's credentials but also the contextual information which may include the traffic nature, the network load as well as the data type. The risk score of a subject $s$ performing an access privileges $a$ on a resource $o$ for a given context $c$ is defined as follows [9]:

$$
\text { Context_Risk }(s, o, a, c)=\sum_{i} w_{i} \cdot r_{i}
$$

Where $w_{i}$ is the weight assigned to metric $i$ and $r_{i}$ is its risk value.

The contextual information incorporating the traffic generation, the data sensitivity and the remote user credentials is defined as follows:

1. Traffic nature: The traffic generated by IoT devices can be categorized into on-demand, emergency, and normal traffic. For instance, in emergency situations where patient's safety is prioritized, the access to the requested data should be granted immediately. Hence, the highest priority is given to emergency traffic [10].

2. Traffic load: When the network is overloaded, the risk of packet loss increases, therefore, the adopted security policy should be set as medium, low or even restricted.

3.Data type: The patient's health records consist of sensitive information such as disease details, family history, and treatments. But other information such as healthy diet and physical exercise is considered as not sensitive.

4. Remote user Credentials: Security policies adopted in the IoT should be adapted to the dynamic context changes where threats and countermeasures could vary in time and space. Therefore, the user's credentials in terms of role, location, and time are incorporated for risk quantification.

\section{B. Security characteristics of access privileges}

Confidentiality, integrity, and availability are the key principles of information security. In fact, adaptive key management and effective encryption while using the appropriate encryption key length reduce the risk of data disclosure. For this purpose, we should consider the effect of user action on the aforementioned security requirements in order to determine the most appropriate security countermeasure. The total risk score for the user's action is given by:
Risk_privileges $(s, o, a, c)=((C P)+(I P)+(A P))$

Where $C, I$, and $A$ present the impact of a user's action on confidentiality, integrity, and availability, respectively, and $P$ is the probability of occurrence of the action.

\section{Quantitative risk evaluation}

The overwhelming priority for remote health monitoring is to make the right decision at the right time. For instance, any loss of data availability can degrade the patient's condition. In addition, confidentiality of the patient's medical records should be protected on the basis of the contextual information. Hence, security countermeasures should be applied while considering the risk level in order to ensure a tradeoff between security effectiveness and network performance. The aggregated risk is defined as:

Aggregated_Risk $(s, o, a, c)=\left(w_{1} \cdot\right.$ Context_Risk $)+\left(w_{2}\right.$. privileges_Risk $)+\left(w_{3} \cdot\right.$ history_Risk $)$

Where Context_Risk and privileges_Risk are obtained by applying (1) and (2), respectively, history_Risk is acquired from the database, and $w_{1}, w_{2}, w_{3}$ are weights attributed to each category of metrics.

\section{STACKELBERG GAME FORMULATION FOR ADAPTIVE REMOTE PATIENT MANAGEMENT: VPN TUNNEL NEGOTIATION}

In this section, we formulate the security policy negotiation among communication peers to establish an authenticated secure tunnel as a game. The main purpose of the proposed approach is to achieve a tradeoff between the security level and the network performance while taking into account the dynamic context changes. Based on the previously presented risk assessment model, the required security policy is identified and its impact on the network performance is determined. In fact, the risk-based decision-making exploits the contextual and historical information to compute the risk score associated with the communicating parties' credentials and capabilities. For instance, when a remote user aims to communicate with the patient or extract information from a body sensor node with a certain QoS (such as delay $\leq 10 \mathrm{~ms}$, bandwidth $\geq 1 \mathrm{Gbit} / \mathrm{s}$, availability $\geq 95 \%$ ) and other technical requirements (security level, service type, etc.), a secure communication channel that supports the above requirements should be established. At first, the remote user initiates the ISAKMP SA negotiation request to the responder VPN. The negotiation request message consists of various transmission suggestions that incorporate different encryption and hash algorithms. Upon receiving the negotiation request message, the responder checks its capacity in terms of battery, computing power, memory size given that some security primitives can be computationally prohibitive for IoT devices. Then, the responder VPN chooses the most appropriate suggestion that fits the communication requirements from the proposals. As a result, the communicating parties agree upon a cryptographic suite. In the following, we firstly define the proposed Stackelberg game. Then, we determine the payoff functions related to the players and we design an adaptive tunnel negotiation algorithm to reach the optimal strategy. 


\section{A. VPN Tunnel Negotiation Game}

In the proposed approach, the negotiation of the cryptographic primitives between the communicating parties is formulated using a leader-follower Stackelberg game. Particularly, a remote user (leader) who wants to extract data from IoT devices has to interact with the trusted IoT gateway (follower) in order to select the most appropriate security policy. For instance, Table I presents the required SA attributes for each security level. The remote user is responsible for announcing QoS requirements and the required security level while considering the contextual information. The follower has to choose adequate parameters from the suggested security primitives defined by the leader. This process requires information about the available resources and energy of the smart things.

\begin{tabular}{|l|l|l|l|l|l|}
\hline $\begin{array}{l}\text { Security } \\
\text { level }\end{array}$ & Encryption & Hash & Mode & Protocol & $\begin{array}{l}\text { Diffie } \\
\text { Hellman } \\
\text { Group }\end{array}$ \\
\hline High & AES & SHA & Transport & ESP & 5 \\
\hline Medium & DES & SHA & Transport & ESP & 2 \\
\hline Low & - & SHA & Transport & AH & 5 \\
\hline Restricted & - & - & - & - & - \\
\hline
\end{tabular}

Table I. Correspondence between security level and security parameters [1]

A Stackelberg game is a non-cooperative game with hierarchical decision-making where players are classified as leaders and followers [11]. The leaders are the initiators of the game who impose their choices and moves on followers. The followers observe the suggestions of leaders and reply with the best response. Furthermore, leaders recognize the above fact and have already proposed their best strategy [12]. Therefore, both leaders and followers reach an agreement to maximize their payoffs. One of the main reasons for applying Stackelberg game is its non-cooperative nature where the leader defines the possible security policies based on the required QoS and the follower has to select one of the proposals while considering its resources availability. In addition, it is a suitable tool for dynamic and distributed environments such as IoT. The remote user and the IoT gateway define their decisions on the basis of their utility functions. Specifically, the negotiation between the players is initiated to reach an agreement on the most appropriate security policy for a given situation with a minimal cost in terms of the network performance. However, the target of the leader is conflicting with the follower. Each player aims to maximize its own utility. On the one hand, the remote user seeks to maximize its payoff by achieving the desired security level with an acceptable QoS. On the other hand, the IoT gateway tries to find the appropriate security level while considering the limited capabilities of the IoT device. The determination of the optimal strategy can be transformed into finding the Stackelberg equilibrium and thus maximizing the profits of the players. The utility functions used in the proposed approach are discussed as follows.

\section{B. Utility Functions}

Based on the above risk assessment model, the decisionmaking of players is dominated by the risk score associated with the contextual information to find out the parameters of the adaptive security policy. On the one hand, the payoff function of the follower depends mainly on the capacity of the selected security policy to resist against security breaches and the required cost in terms of communication overhead, latency, and memory. On the other hand, the utility function of the leader represents the impact of the adopted security policy on the cost in terms of QoS performance. Specifically, the multidimensional utility function defines the cost of implementing a security policy on the network performance and the impact of VPN configuration on security policy violation. We consider a damage function, denoted by $\Delta$, which returns the efficiency of the VPN configuration, and a network utility function, $\Lambda$, which determines the impact of the security agreement on the performance degradation in terms of latency, computational cost, communication overhead. We adopt the sigmoid function thanks to its efficiency to adjust utilities according to the different requirements [13]. The utility functions are defined as follows:

$$
\begin{gathered}
\Delta\left(P_{p v}\right)=\left(1+e^{-g_{p v} \cdot\left(P_{p v}-h_{p v}\right)}\right)^{-1} \\
\Lambda\left(P_{p d}\right)=1-\left(1+e^{-g_{p d}\left(P_{p d}-h_{p d}\right)}\right)^{-1}
\end{gathered}
$$

Where $P_{p v}$ and $P_{p d}$ are the probabilities of security policy violation and performance degradation, $g_{p v}, g_{p d}$ represent the sensitivity of the utility functions, and $h_{p v}, h_{p d}$ determine the inflection points.

A security policy violation occurs when a VPN tunnel is misconfigured and a malware succeeds to modify stealthy the VPN configuration. Performance degradation is related to the cost of security policy implementation into the network performance in terms of latency, throughput, packet loss, and energy efficiency. The utility functions presented above express a trade-off between the security policy (at the risk of depleting the IoT device battery, increasing delays and packet loss rate, and decreasing throughput) and network performance (at the risk of security policy breaches). Based on this trade-off, we formulate a multilateral Nash Bargaining model where Nash equilibrium can be determined so that utilities are maximized. The players execute random strategies to reach an agreement and release a balance between high performance and stronger security.

Solving the following payoff function defines the Nash equilibrium which is denoted by $\left(\Delta^{*}, \Lambda^{*}\right)$ :

$$
U^{t}=\max \left(1-\Delta\left(P_{p v}\right)\right) \cdot \Lambda\left(P_{p d}\right)
$$

The existence of the Nash equilibrium for the proposed game is justified by the fact that the aforementioned objective function (equation (5)) is continuous and defined on a compact. The equilibrium solution requires the computation of the probabilities $P_{p v}$ and $P_{p d}$ as follows:

We denote by $\mathrm{A}=\left(a_{i, j}\right)$ transition matrix that represents the transition probabilities between the previous state where a VPN 
configuration $i$ is performed and the current state where a VPN configuration $j$ is proposed

$$
\begin{aligned}
& P_{p v}=\frac{\pi(\mu, i)}{\sum_{j} \pi(\mu, j)} \\
& P_{p d}=\sum_{j} \pi(S, i)
\end{aligned}
$$

Where $S$ defines the network status for the configuration $i ; \mu$ is the marking place where the VPN is breached according to the Stochastic Activity Network (SAN) terminology [14][15]; $\pi$ is the steady state probability matrix, obtained through the resolution of the equations $\pi . A=\pi$ and $\pi . \mathbb{1}=\mathbb{1}$, where $\mathbb{1}$ is a matrix of ones. More details about the probabilities computation can be found in [8][14].

\section{Adaptive Negotiation Algorithm}

The major aspects related to adaptive security management are QoS satisfaction, energy efficiency, and security effectiveness. For this purpose, Algorithm 1 has been designed to efficiently establish a secure tunnel between the remote user and the IoT device while ensuring an acceptable network performance. The first move is for the leader, i.e., the remote user who aims to communicate securely with a high QoS satisfaction with the IoT device. Once the follower receives the communication request, it evaluates the risk score and the network status in order to choose a suitable suggested security policy. Based on the utility function the IoT gateway chooses the optimal VPN configuration.

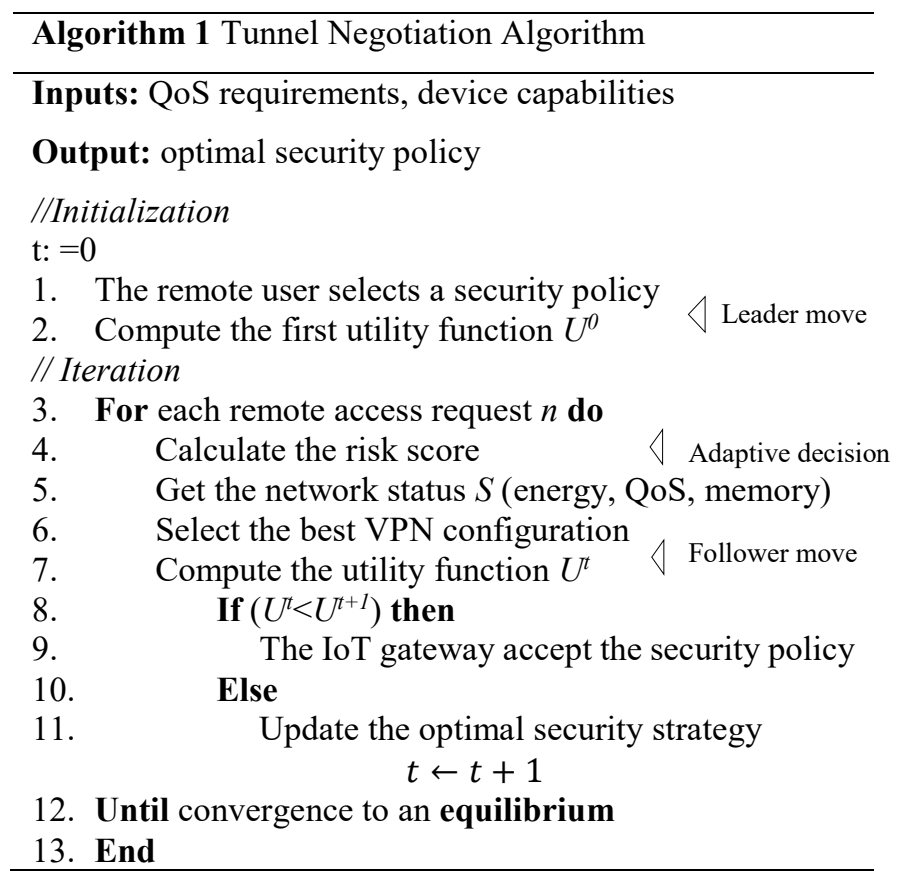

\section{PERFORMANCE EVALUATION}

In this section, we evaluate the performance of the gamebased adaptive security management approach. VPN Security policy violation is dependent on many factors such as malware infection, maintenance frequency, and traffic load. Our main purpose is to find the most appropriate VPN configuration that ensures an acceptable security level without affecting the network performance in terms of resource consumption and latency. In fact, we study the impact of adaptive security decision on the performance degradation. We consider the secure tunnel negotiation process on the basis of the Stackelberg game for a teleconsultation application to evaluate the behavior of the proposed model compared with static security scenarios where high security level (ESP+AES+SHA configuration) or medium security level (ESP+DES+SHA) are continuously performed. The communication and computational cost of the authentication and encryption algorithms are given in [16][17]. The main simulation parameters are summarized in Table II

\begin{tabular}{|l|l|}
\hline Parameter & Value \\
\hline End-to-end delay threshold & $150 \mathrm{~ms}$ \\
Data size & $8-6500$ bytes \\
Data rate & $20 \mathrm{Mb} / \mathrm{s}$ \\
IoT device processing capacity & $100 \mathrm{MIPS}$ \\
\hline
\end{tabular}

Table II. Simulation parameters

At first, we perform the evaluation of the network performance in terms of communication overhead as a function of the traffic load. In fact, we study the effect of the padding procedure on packet size increase during the authentication and encryption process. In Figure 4, we present the communication overhead which is related to the transmitted data that highly affect the energy consumption of the IoT device. For an adaptive security policy where the security level may decrease or increase dynamically on the basis of the risk score the communication cost is improved by $27 \%$ compared to high security decision. For instance, if the risk score exceeds $75 \%$ high security level should be applied. However, when the risk score is lower than $25 \%$ no security checks are performed. Thus adaptive security decision achieves better performance in terms of communication cost compared to static security decision where high or medium security level is permanently implemented.

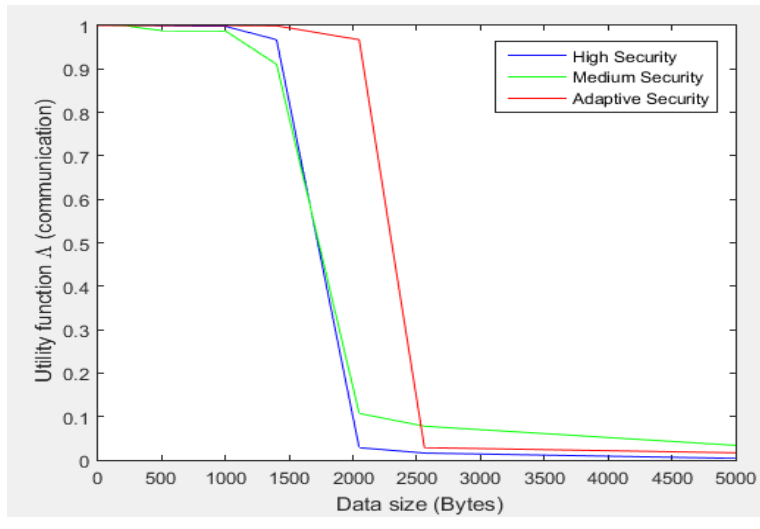

Figure 4. The impact of security policy selection on the communication overhead

To investigate the impact of the proposed adaptive security approach on the system performance in terms of latency for a sensitive delay application where the latency shouldn't exceed $150 \mathrm{~ms}$, we performed the simulation for different data sizes and a fixed processor capacity. As shown in Figure 5, the network performance achieved through our game-based approach outperforms both permanent high security and medium security 
decisions. In fact, it is found that non-adaptive security scenario where high security level is permanently deployed regardless the contextual information hardly affects the system performance. For the adaptive decision, if the traffic load is heavy the security level should be decreased to avoid the system overload and provide the required QoS (latency) for a sensitive delay application. If the risk level exceeds a predefined threshold the security level should be increased.

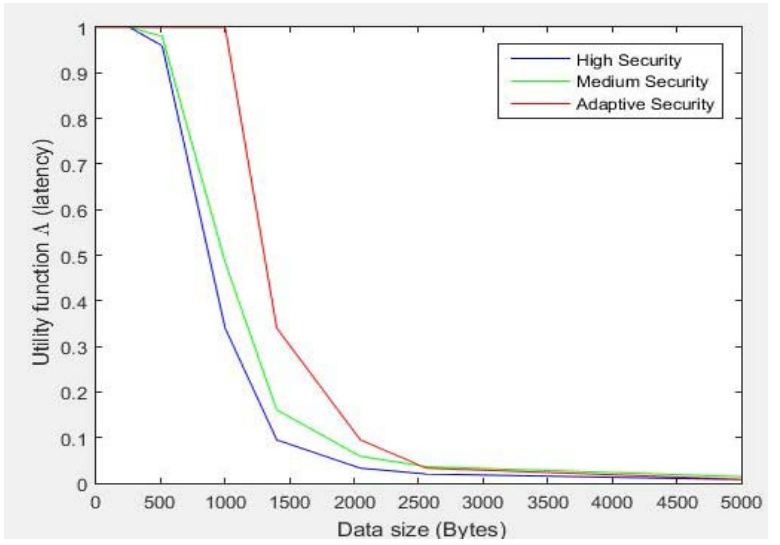

Figure 5. Performance degradation in terms of latency for different data sizes

Finally, Figure 6 illustrates the evolution of the computational cost as a function of different data sizes. In the case where adaptive negotiation decision is made, the performance degradation increases slowly when data size increases. This is because the security level is decreased for heavy traffic load. The average improvement of the proposed game theoretic approach is around $25 \%$ compared to a static security decision.

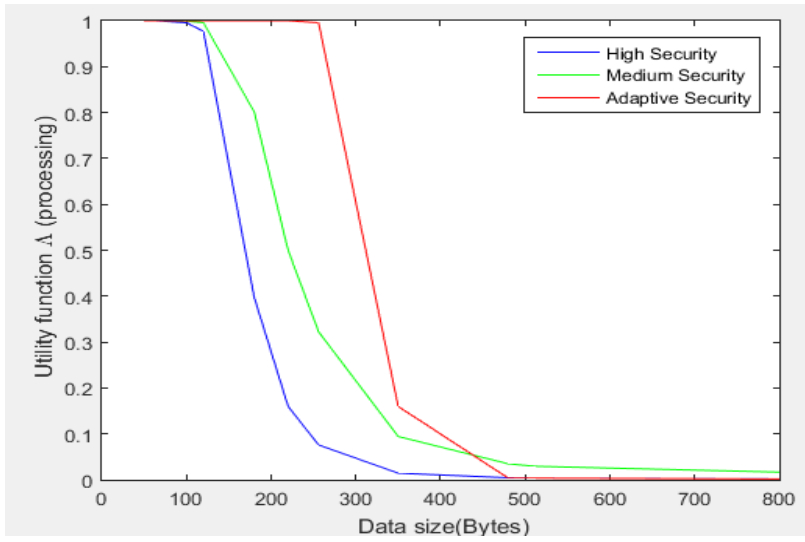

Figure 6 . The computational cost of different security policies

As a result, the adaptive VPN configuration where the security policy is selected on the basis of the risk score and the IoT device capabilities enhances the network performance in terms of resource allocation and QoS satisfaction (delay) while dynamically adjusting the security parameters (authentication and encryption protocols). In fact, if the risk score exceeds $75 \%$ the more efficient VPN configuration should be implemented. However, low security policy may be performed for a risk score lower than $50 \%$.

\section{CONCLUSION}

In this paper, we addressed the adaptive security for VPN tunnel negotiation in order to guarantee a tradeoff between the security effectiveness and the network performance. In our model, the VPN configuration is performed while considering the dynamic context changes and the devices' capabilities. In this context, we proposed a Stackelberg game between a remote user and an IoT gateway to negotiate the security parameters and thus select the most appropriate security policy. Then, we evaluated the cost of the adopted VPN configuration in terms of performance degradation. We demonstrated the efficiency of our proposed approach as compared to static security decision.

\section{REFERENCES}

[1] A. Bahnasse, F. Louhab, M.Talea, \& al.” Towards a New Approach for Adaptive Security Management in New Generation Virtual Private Networks", International Conference on Wireless Networks and Mobile Communications(WINCOM), 2017.

[2] Sheltami TR, Bala A, Shakshuki EM ,'Wireless sensor networks for leak detection in pipelines: a survey". J Ambient Intell Humaniz Comput 7(3):347356,2016

[3] Jay Bryan R. Lawas, Allan C. Vivero, Ankit Sharma, "Network Performance Evaluation of VPN Protocols (SSTP and IKEv2)", International Conference on Wireless and Optical Communications Networks (WOCN), 2016

[4] S. Jahan, Md. Rahman, S. Saha," Application Specific Tunneling Protocol Selection for Virtual Private Networks", International Conference on Networking, Systems and Security NSysS, 2017.

[5] S. Fluhrer, "System and method for encrypted group network communication with point-to-point privacy.", U.S. Patent No. 8,160,255. 17 Apr. 2012.

[6] W.J. Francis, and D. McAloon. "Real-time automated virtual private network (VPN) access management.", U.S. Patent No. 9,571,352, 14 February 2017.

[7] M. Hamdi, H. Abie, "Game-Based Adaptive Security in the Internet of Things for eHealth", IEEE International Conference on Communications, June.2014.

[8] A. Arfaoui, A. Ben Letaifa, A. Kribeche SM. Senouci M. Hamdi, "A Stochastic Game for Adaptive Security in Constrained Wireless Body Area Networks", IEEE CCNC2018, Las Vegas, USA, 12-15 January, 2018.

[9] D. R. Santosa, R. Marinhoa, G. R. Schmitta, C. M. Westphalla, “A Framework and Risk Assessment Approaches for Risk-based Access Control in the Cloud", Journal of Network and Computer Application, Vol. 74, 2016

[10] I. Anjum, N. Alam, M. A. Razzaque, M. Mehedi Hassan, and A. Alamri, "Traffic priority and load adaptive MAC protocol for QoS provisioning in body sensor networks," International Journal of Distributed Sensor Networks, 2013.

[11] P. Y. Nie and P. A. Zhang, "A note on stackelberg games," in Chinese Control and Decision Conference, 2008, pp. 1201-1203.

[12] J. Lee, J. Guo, J. K. Choi, and M. Zukerman, "Distributed energy trading in microgrids: A game-theoretic model and its equilibrium analysis," IEEE Transactions on Industrial Electronics, vol. 62, no. 6, pp. 3524-3533, Jun 2015 .

[13] Chungang Yang, Jiandong Li, Waleed Ejaz, Alagan Anpalagan Mohsen Guizani,"State Utility function design for strategic radio resource management games: An overview, taxonomy, and research challenges", Transactions on Emerging Telecommunications Technologies Journal, May.2016

[14] S. Rahimi, M. Zargham. "Security Analysis of VPN Configurations in Industria Control Environments", 5th International Conference Critical Infrastructure Protection (ICCIP), Mar 2011

[15] S. Maza." Stochastic activity networks for the performance evaluation of fault tolerant systems", QUALITA 2013, Mar 2013

[16] Ch. Xenakis, L. Merakos, "IPsec-based end-to-end VPN deployment over UMTS", J. computer communication, 27(17): 1693-1708, 2004

[17] O. Elkeelany, M. M. Matalgah, K. P.Sheikh, \& al., "Performance Analysis of IPSec Protocol: Encryption and Authentication", IEEE International Conference on Communications (ICC), 2002 